\title{
STABILITY OF ASTEROIDAL MOTION \\ IN THE HECUBA GAP
}

\author{
H. SCHOLL and R. GIFFEN \\ Astronomisches Rechen-Institut, Heidelberg, Germany
}

There exist gaps in the frequency distribution of the osculating mean motions of the asteroids. In these gaps, the mean motions of the asteroids are commensurable to Jupiter's mean motion.

Recently, Giffen (1973) studied the stability of commensurable motion in the Hecuba gap where the ratio of the asteroid's mean motion to that of Jupiter is $2: 1$. Giffen's model was based on the plane, elliptic restricted three-body problem which means that $e_{A}, e_{J} \neq 0, i_{A}=i_{J}=0$ and $m_{A}=0$. The dimension of the corresponding phase space is 5 . Poincaré developed a system of canonical variables that are suitable for the study of the secular behaviour of commensurable motion in the elliptic restricted three-body problem. This system was then modified slightly by Schubart. The Hamiltonian $H(\ldots t)$ of this system depends on the mean anomaly which is a function of the time. If one removes that part of the Hamiltonian $H$ which depends on the time then the new Hamiltonian $H^{*}$ is constant and therefore is a nonclassical integral of the motion. Schubart obtained such a nonclassical integral by averaging the Hamiltonian $H$ over the commensurable period. The resulting averaged Hamiltonian $H^{*}$ depends then on four variables, that is, $H^{*}=H^{*}(a, e, \sigma, g)$, where $\sigma$ is the critical argument. For a given value of $H^{*}$, the motion of an asteroid can be described in a three-dimensional phase space, $H^{*}(a, e, \sigma)$.

To study the stability of the motion in the Hecuba gap, Giffen used the method of invariant curves. He chose a certain plane in the three-dimensional phase space. Each time when the orbit crosses this plane, the point of intersection is plotted. Figure 1 shows these plotted points for an orbit with $e=0.30$. The points are plotted when the eccentricity is close to its minimum. All the points lie on a curve. That means that each time when the orbit in the phase space crosses the designated plane, the intersection point will lie on this invariant curve. Therefore, apparently, for this orbit there exists an isolating integral which indicates that the motion of an asteroid in the Hecuba gap with eccentricity $e=0.30$ is stable. Giffen found that all the other high eccentric orbits he computed have invariant curves.

In Figure 2, points for a low eccentric orbit, $e=0.14$, are plotted. The points do not lie on an invariant curve. They have the tendency to fill the whole area. This orbit is 'ergodic'. Here and in the following 'ergodic orbits' mean 'wild' orbits. It is not stated that the orbits are ergodic in the exact mathematical sense.

More examples show that the small eccentric orbits in the Hecuba gap are all ergodic. 140 points are plotted here which corresponds to a run over $60000 \mathrm{yr}$. The points of the first $30000 \mathrm{yr}$ fall in the area on the left side and the points of the second $30000 \mathrm{yr}$ fall in the area on the right side of Figure 2. This indicates that on the aver-

Y. Kozai (ed). The Stability of the Solar System and of Small Stellar Systems, 77-79. All Rights Reserved Copyright $\mathrm{C} 1974$ by the IAU. 


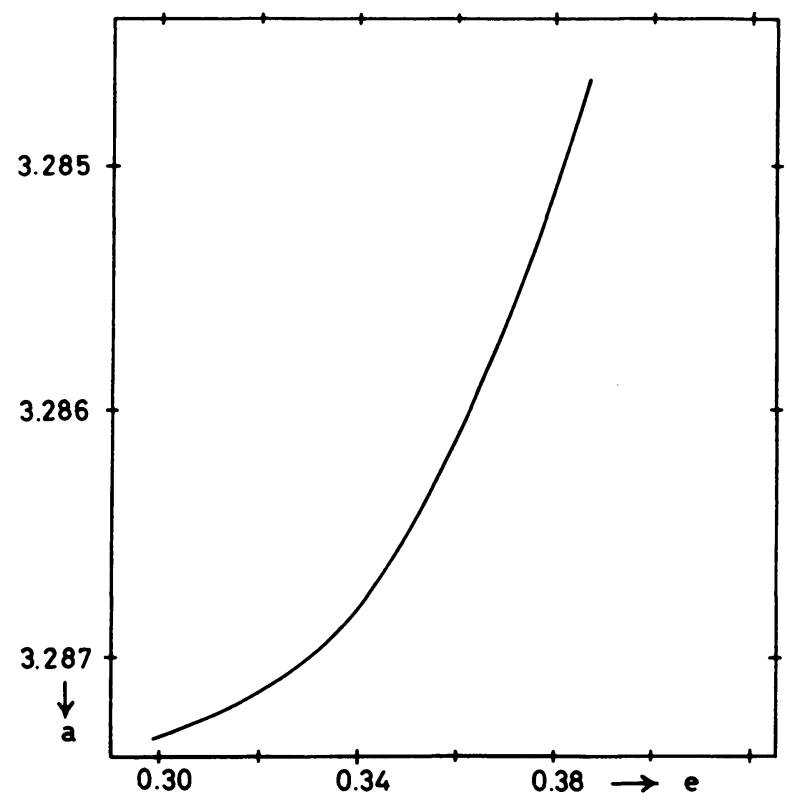

Fig. 1. Plots of intersections of orbits with a plane in the phase space, where the eccentricity is close to its minimum $(e=0.30)$.

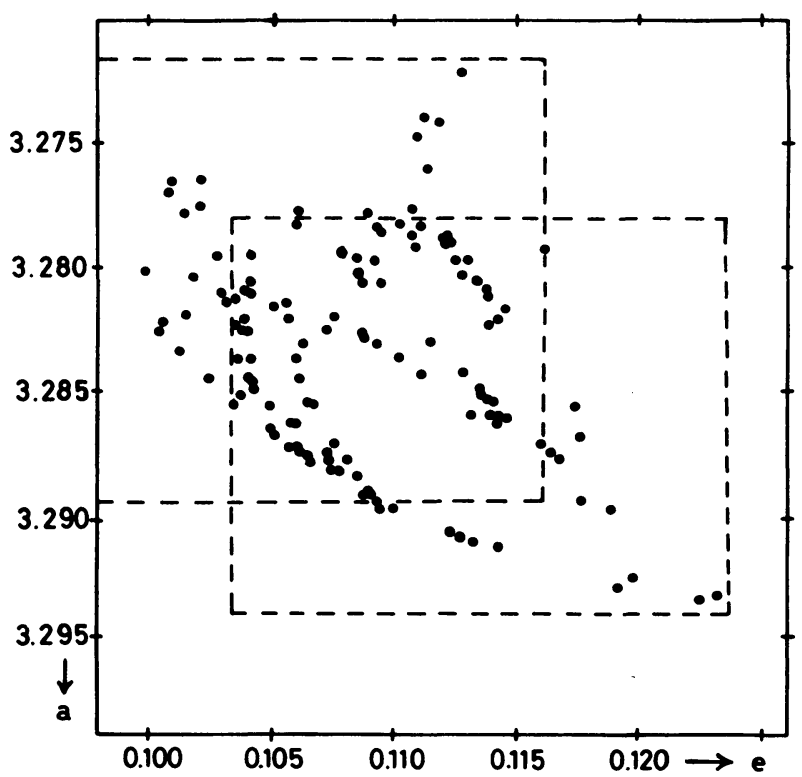

Fig. 2. Plots of intersections of orbits with a plane for $e=0.14$. 
age the eccentricity increases. This fact leads Giffen to the following hypothesis: If there was an asteroid with small eccentricity in the Hecuba gap, then its eccentricity increased on the average. If the eccentricity was large, the collision probability with asteroids close to the Hecuba gap became larger, so that the asteroid left the Hecuba gap after collisions. This procedure could explain why there are no asteroids with small eccentricity in the Hecuba gap.

To test this hypothesis, we made computations over a longer period of time, over 200000 yr instead of 60000 yr. The results show that for the high eccentric orbits the invariant curves do not dissolve. For small eccentric orbits, the eccentricity increases but then decreases. On the average, it oscillates. No slow increase on the average in the eccentricity was found.

In addition, according to a suggestion from Froeschlé, we studied the divergence of orbits which start with slightly different initial values. The high eccentric orbits diverge very slowly while the small eccentric orbits diverge quickly. This result also indicates that the small eccentric orbits are ergodic and the high eccentric orbits are nonergodic. However, after $200000 \mathrm{yr}$, the eccentricity of the ergodic orbits did not increase as was expected by the hypothesis. But this is not yet a disproof of the hypotheses because it is possible that the small eccentric orbits develop by a diffusion process, which has been described by Froeschlé and Scheidecker (1974), into higher eccentric orbits and then escape from the gap because of collisions. In collaboration with Froeschle we now are testing this new hypothesis and we also are testing if the invariant curves for the high eccentric orbits dissolve very slowly and become therefore also ergodic.

If there were asteroids with small eccentric orbits in the Hecuba gap and if these asteroids left the gap because of perturbations of Jupiter which increased their eccentricities so that collisions occured, then this process must have taken a much longer time than $10^{5} \mathrm{yr}$.

\section{Acknowledgements}

The authors would like to express their gratitude to Dr J. Schubart, Astronomisches Rechen-Institut Heidelberg, for his helpful advice and for many valuable discussions. The calculations were carried out on the IBM 360/44 of the University of Heidelberg's Rechenzentrum.

\section{References}

Froeschlé, C. and Scheidecker, J.-P.: 1974, this volume, p. 297.

Giffen, R. B.: 1973, Astron. Astrophys. 23, 387-403. 